Meta

Journal des traducteurs

Translators' Journal

\title{
Traduction de la littérature française au Brésil : état de la question
}

\section{Marie-Hélène C. Torres}

Volume 48, numéro 4, décembre 2003

URI : https://id.erudit.org/iderudit/008722ar

DOI : https://doi.org/10.7202/008722ar

Aller au sommaire du numéro

\section{Éditeur(s)}

Les Presses de l'Université de Montréal

ISSN

0026-0452 (imprimé)

1492-1421 (numérique)

Découvrir la revue

Citer cet article

Torres, M.-H. C. (2003). Traduction de la littérature française au Brésil : état de la question. Meta, 48(4), 498-508. https://doi.org/10.7202/008722ar

\section{Résumé de l'article}

Cet article propose de décrire la position de la littérature de langue française traduite en portugais dans le système culturel et littéraire brésilien. Après avoir retracé les liens historiques d'attirance mutuelle entre le Brésil et la France, politiquement et économiquement parlant mais également culturellement, j'analyse les données de la littérature traduite dans le monde et de langue française au Brésil à partir du corpus de l'Index Translationum de l'Unesco. Finalement, quelques stratégies sont avancées pour relancer la traduction d'oeuvres littéraires françaises et de langue française au Brésil dont le volume est encore faible face aux traductions issues de l'anglais. 


\title{
Traduction de la littérature française au Brésil: état de la question'
}

\author{
MARIE-HÉLÈNE C. TORRES \\ Université fédérale de Santa Catarina, Florianópolis, Brésil \\ marie@cce.ufsc.br
}

\begin{abstract}
RÉSUMÉ
Cet article propose de décrire la position de la littérature de langue française traduite en portugais dans le système culturel et littéraire brésilien. Après avoir retracé les liens historiques d'attirance mutuelle entre le Brésil et la France, politiquement et économiquement parlant mais également culturellement, j'analyse les données de la littérature traduite dans le monde et de langue française au Brésil à partir du corpus de l'Index Translationum de l'Unesco. Finalement, quelques stratégies sont avancées pour relancer la traduction d'œuvres littéraires françaises et de langue française au Brésil dont le volume est encore faible face aux traductions issues de l'anglais.
\end{abstract}

\begin{abstract}
This paper sets out to describe the position in Brazilian cultural and literary system of French language literature translated into Portuguese. After considering the historical relations of mutual attraction between Brazil and France, politically, economically and culturally, data on translated literature at a world level are analysed, and of French language in Brazil in particular as they emerge from the UNESCO Index Translationum. Finally, some strategies are suggested as to the republishing in Brazil of French literature translations, still very modest in number when compared to translations from the English.
\end{abstract}

\section{MOTS-CLÉS/KEYWORDS}

Index Translationum, traduction littéraire, Brésil, analyse de données

La traduction, principalement aux prémices du $\mathrm{xxI}^{\mathrm{e}}$ siècle, est intégrée à tous les niveaux de la société et s'est rendue indispensable au fonctionnement de celle-ci. Certains auteurs parlent même volontiers aujourd'hui de l'ubiquité de la traduction, visible et invisible ou masquée. La traduction a notamment acquis une fonction, nous dit José Lambert (1989: 215), celle de la communication, ou plutôt, ajoute-t-il, de l'internationalisation de la communication, car elle est un acte de communication qui établit une relation interactive entre les cultures (Lambert 1995: 20). Effectivement, la traduction, et d'autant plus la traduction littéraire qui est l'objet qui nous occupe aujourd'hui, ou mieux, la littérature traduite, a non seulement une fonction communicative mais encore culturelle et interculturelle, comme le constate Anthony Pym (1998: 1991).

Partant donc de l'hypothèse de Lambert selon laquelle la traduction établit une relation interactive entre les cultures ou, en d'autres termes, de celle de l'interculture de $\mathrm{Pym}^{2}$, nous essayons de décrire la position de la littérature de langue française traduite en portugais dans le système culturel et littéraire brésilien. Outre des raisons d'ordre personnel $^{3}$, notre intérêt pour la littérature française traduite en portugais s'explique à plusieurs titres. Nous nous contenterons ici d'évoquer simplement ces 
raisons. D'abord, nous avons noté un volume assez faible de traductions en portugais des livres français (tous domaines confondus) par rapport à des livres issus d'autres langues (comme l'anglais par exemple). Ensuite, nous avons relevé que des liens spécifiques unissaient le Brésil et la France, non seulement des liens historiques et coloniaux - la France a tenté à plusieurs reprises de coloniser le Brésil, comme nous le verrons - mais encore des liens d'amitié et de coopération intellectuelle, scientifique et culturelle - souvent académique - depuis les prémices du $\mathrm{xx}^{\mathrm{e}}$ siècle. Enfin, nous avons remarqué que la littérature brésilienne occupe une place paradoxale dans ce que Pascale Casanova appelle «La république mondiale des lettres». Précisons qu'avant Casanova, dès 1990, José Lambert se référait déjà à ce qu’il a nommé «les cartes mondiales de la littérature », établies selon le principe de la multiplication des cartes qui pourrait ainsi «renouveler et réorienter notre présentation littéraire de l'univers ». Selon Casanova (1999), il existe « un méridien de Greenwich » de l'univers littéraire mondial dont Paris serait la capitale et «auquel se mesurent la nouveauté et la modernité des œuvres». Le Brésil ne fait pas partie de ce méridien de Greenwich et sa littérature consiste en ce que Casanova nomme «petites littératures » par opposition aux «littératures dominantes», ce qui expliquerait le faible volume de traduction au Brésil et peut-être le manque d'intérêt de la part des politiques éditoriales étrangères à y exporter leur littérature.

Mais si la traduction, comme l'affirme Casanova, « est la grande instance de consécration spécifique de l'univers littéraire», pourquoi la littérature de langue française et française - littérature centrale de l'univers littéraire mondial - n'est-elle pas plus traduite au Brésil?

\section{LE PASSÉ}

Si nous examinons le passé (pour utiliser le titre de notre congrès), nous pouvons voir qu'une longue histoire d'attirance mutuelle unit le Brésil et la France, politiquement et économiquement parlant mais également culturellement.

Selon la tradition dieppoise, la découverte du Brésil serait attribuée à Jean Cousin, quatre ans avant le premier voyage de Colomb. Lévi-Strauss (1955: 88) ajoute qu' «à moins d'un miracle, le problème ne sera jamais résolu puisque les archives dieppoises, y compris la relation de Cousin, ont disparu au XVII ${ }^{\mathrm{e}}$ siècle au cours de l'incendie dû au bombardement anglais» (1955: 89). Dans le même sens, Carelli', qui était responsable de la banque de données France/Brésil au CNRS, affirme également que le Brésil n'était pas inconnu des marins français de l'époque et que dès 1504, «le capitaine Binot Paulmier de Gonneville [sic] aborda nombre d'expéditions s'ensuivit, surtout de marins normands en quête de bois de braise (pau-brasil) dont le commerce était très actif à Rouen au milieu du XVI ${ }^{\mathrm{e}}$ siècle ». Au cours de ces expéditions, des truchements français étaient laissés au Brésil afin d'apprendre les langues indiennes pour servir à leur tour d'interprètes à leurs compatriotes.

Sous la poigne de Nicolas Durand de Villegagnon, qui voulait fonder une colonie au Brésil, fut donc établie, dans la baie de Guanabara à Rio de Janeiro, la France Antarctique en 1555. Mais ce rêve ne dura que peu de temps puisque ce « refuge pour les protestants persécutés qui voulaient quitter la métropole» (Lévi-Strauss 1955: 88) se désagrégea à la suite de longues disputes avec les catholiques également représentés. Les Portugais n'eurent donc pas de mal à reprendre possession du fort français. 
Et donc, en 1560, les Français furent expulsés, leur erreur ayant peut-être été d'essayer de reproduire le vieux monde et ses références familières dans le nouveau, notamment les guerres de religion. En 1612 toutefois, nous dit Carelli (1993: 47), une expédition de nobles, d'hommes d'armes et de religieux capucins fondèrent la France Equinoxiale à Saint-Louis-du-Maragnon. Ils essayèrent en vain d'intéresser la cour de France ainsi que de riches marchands à leur expédition. Ce fut peine perdue puisque dès 1615, le fort Saint-Louis était tombé aux mains des Portugais. Ces velléités coloniales avortées s'effacèrent des mémoires, mais les rapports entre la France et le Brésil allaient prendre une tout autre tournure.

Ce sera grâce au Roi D. João VI, installé avec la cour portugaise à Rio de Janeiro, que les rapports entre le Brésil et la France s'intensifièrent. Il fit en effet «venir pour la fondation d'une Académie des beaux-arts sous les tropiques une mission d'artistes français» (Carelli 1987: 131), en 1816, composée de peintres, sculpteurs, architectes, graveurs, ingénieurs... Leur influence fut déterminante pour l'évolution des arts et provoqua la venue de nombreux français d'horizons divers - boulangers, pâtissiers, cuisiniers, orfèvres, professeurs de musique, de français... - qui s'installèrent au Brésil. Les mœurs en furent également touchées et il était de bon ton d'envoyer les jeunes Brésiliens étudier dans les universités françaises. D’ailleurs, dans le pays, la langue, la littérature et la mode françaises étaient à leur apogée. L'envoi considérable de livres français vers le Brésil aura un impact énorme sur l'évolution des mentalités, affirme Carelli. Ce commerce du livre se développera - après avoir été interdit, rappelons-le jusqu'en 1808 - notamment grâce à Baptiste-Louis Garnier, installé depuis 1844 à Rio de Janeiro, où il mourut en 1893 (Carelli 1987: 134). Les Brésiliens lisaient donc en français, ce qui freine les traductions bien évidemment. Le Brésil du $\mathrm{xx}^{\mathrm{e}}$ siècle se détachera progressivement du modèle français par une émancipation culturelle et identitaire - surtout propulsée par les modernistes. La séduction primaire se métamorphosait en relations d'échange, de coopération et d'hommages. Les voyageurs français, entre autres Anatole France, Darius Milhaud, Benjamin Péret ou encore Blaise Cendrars pour les plus célèbres, favorisèrent une (re)découverte du Brésil. Et dans l'espoir de divulguer la culture française, fut envoyé à Rio de Janeiro en 1908 Georges Dumas en tant que porte-parole du «Groupement des Universités et Grandes Ecoles de France» pour mettre sur pied la coopération avec le Brésil (Carelli 1987: 157). D’où il s'ensuivit une série de conférences à la Sorbonne, le 3 avril 1909, d'Anatole France, Victor Orban et Oliveira Lima", sous le nom de «Fête de l'intellectualité brésilienne» dont le but principal était de rendre hommage à l'écrivain Machado de Assis, décédé l'année précédente, et de diffuser ses œuvres en France (Valezi Staut 1991: 14). L'on y fera pour l'occasion l'apologie des liens de la latinité qui uniraient les deux pays.

Cette fête aura permis un raffermissement de la coopération universitaire mutuelle avec, d'un côté, la création en 1911 d'une chaire d'études brésiliennes à la Sorbonne et plus tard de l'Institut des Hautes Études de l'Amérique latine à Paris, et de l'autre côté, l'envoi d'une mission d'universitaires français en 1934 dont Claude Lévi-Strauss ${ }^{6}$ qui inaugurèrent les cours de la USP, l'Université de São Paulo. Peu à peu, le Brésil gagnait une identité en France, à travers non seulement l'Académie mais encore les encyclopédies et revues, divulgatrices d'une «re-présentation» du Brésil, selon l'expression de Niranjana. 


\section{LE PRÉSENT : analyse des données de la littérature traduite dans le monde et de langue française au Brésil}

Nous proposons ici de faire un compte rendu sommaire de nos recherches actuelles sur la littérature traduite au Brésil. En effet, le manque d'études plus systématiques sur l'histoire et la critique de la traduction ainsi que la constatation qu'il n'y pas encore au Brésil de bibliographie des traductions brésiliennes d'œuvres littéraires ont servi de point de départ à un projet intégré, duquel je fais partie, un projet intitulé «Traduction, tradition et innovation: le rôle des traductions de l'allemand, de l'espagnol, du français et de l'italien (1970-2000) au Brésil». Il nous semble fondamental de situer et de décrire en chiffres la traduction d'œuvres littéraires de langue française afin de sensibiliser les autorités compétentes sur le fait que la diffusion de la langue et de la culture de langue française au moyen des traductions n'a pas actuellement la visibilité qu'elle avait par le passé car aujourd'hui le public-lecteur brésilien ne lit plus en français comme par le passé.

N'ayant donc pas à notre disposition de banques de données d'œuvres traduites au Brésil, nous avons commencé nos recherches avec le corpus en ligne de l'Index Translationum de l'Unesco. Comme les statistiques disponibles sont souvent embryonnaires et relatives - car les cessions de titres ne sont pas toujours relevées à la source et leur enregistrement par le service de dépôt légal des différentes bibliothèques nationales ne présente aucune homogénéité - l'Index Translationum de l'Unesco reproduit le retard de saisie dans les données transmises par les bibliothèques nationales. Les chiffres recueillis permettent néanmoins de dégager de grandes tendances. Il nous faudra bien sûr par la suite compléter ces données.

Passons donc aux données statistiques. Pour la première étape, nous avons d'abord cherché à savoir combien de titres ont été traduits dans le monde à partir de chaque langue de 1970 à 2003 (tableau 1).

TABLEAU 1

Volume de titres des langues les plus traduites du monde (tous domaines confondus)

\begin{tabular}{|c|c|c|c|}
\hline Langues & Volume & $\begin{array}{l}\text { Taxinomie des langues } \\
\text { les plus traduites } \\
\text { dans le monde }\end{array}$ & Pourcentage \\
\hline Anglais & 688945 & 1 & $52 \%$ \\
\hline Français & 137030 & 2 & $10 \%$ \\
\hline Allemand & 119720 & 3 & $9 \%$ \\
\hline Russe & 85669 & 4 & $7 \%$ \\
\hline $\begin{array}{l}\text { Scandinaves (suédois, danois, } \\
\text { finnois, norvégien) }\end{array}$ & 42969 & 5 & $3 \%$ \\
\hline Italien & 39169 & 6 & $3 \%$ \\
\hline Espagnol & 29004 & 7 & $2 \%$ \\
\hline Tchèque & 11569 & 8 & $1 \%$ \\
\hline Hollandais & 10971 & 9 & $1 \%$ \\
\hline Polonais & 9554 & 10 & $1 \%$ \\
\hline Autres Langues ${ }^{7}$ & 139743 & & $11 \%$ \\
\hline $\begin{array}{l}\text { Total du volume des } 10 \text { langues } \\
\text { les plus traduites dans le monde }\end{array}$ & 1174600 & & $\begin{array}{c}89 \% \\
\Rightarrow \text { Pourcentage par rapport } \\
\text { au total des traductions } \\
\text { de } 1970 \text { à } 2003\end{array}$ \\
\hline
\end{tabular}


L'anglais est la langue à partir de laquelle on traduit le plus, c'est-à-dire que c'est la langue la plus traduite du monde de 1970 à 2003, avec la moitié des traductions.

Les titres traduits du français, de l'allemand et du russe oscillent entre $7 \%$ et $10 \%$ du total. Ces données sont encore plus parlantes si on les reporte sur un graphique.

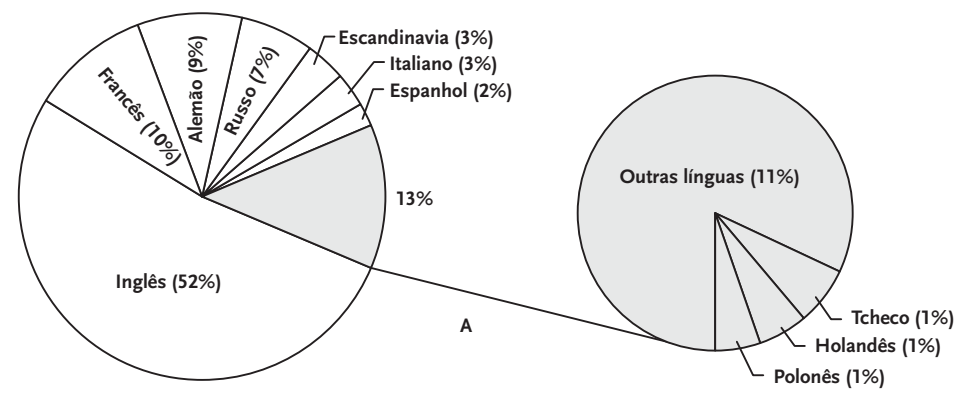

Donc, les titres traduits du français, de l'allemand et du russe représentent dans leur ensemble un quart des traductions produites dans le monde de 1970 à 2003.

Nous observons également que les dix langues les plus traduites représentent $89 \%$ du volume total des traductions, contre $11 \%$ distribués en une dizaine d'autres langues dont le portugais. Ces chiffres montrent bien que le portugais ne fait pas partie du palmarès des langues les plus traduites.

En ce qui concerne la littérature, ce qui nous intéresse à présent est de savoir quel est le volume de titres en littérature qui ont été traduits dans le monde et à partir de quelle langue entre 1970 et 2003, afin de découvrir quelles sont les littératures les plus traduites dans le monde (tableau 2).

TABLEAU 2

Volume de traduction littéraire dans le monde $=645671$ titres

\begin{tabular}{|l|c|c|c|}
\hline Langues & Volume & $\begin{array}{c}\text { Taxinomie des langues } \\
\text { les plus traduites } \\
\text { dans le monde }\end{array}$ & Pourcentage \\
\hline Anglais & 366548 & 1 & $58 \%$ \\
\hline Français & 65496 & 2 & $10 \%$ \\
\hline Allemand & 44741 & 3 & $7 \%$ \\
\hline Russe & 28354 & 4 & $4 \%$ \\
\hline $\begin{array}{l}\text { Scandinaves (suédois, danois, } \\
\text { finnois, norvégien) }\end{array}$ & 26988 & 5 & $4 \%$ \\
\hline Italien & 16055 & 6 & $2 \%$ \\
\hline Espagnol & 14991 & 7 & $1 \%$ \\
\hline Polonais & 4928 & 8 & $1 \%$ \\
\hline Néerlandais & 4906 & 9 & $1 \%$ \\
\hline Tchèque & 4465 & 10 & $1 \%$ \\
\hline Japonais & 4416 & 11 & $8 \%$ \\
\hline Portugais & 3581 & 12 & $100 \%$ \\
\hline Autres langues & 52280 & & $2 \%$ \\
\hline TOTAL & 645671 & & \\
\hline
\end{tabular}


Les littératures de langue anglaise sont les plus traduites avec $58 \%$, bien devant la littérature de langue française, qui représente seulement $10 \%$ du total de la littérature traduite dans le monde, ou celle de langue allemande avec $7 \%$ ( $3^{\mathrm{e}}$ place), comme nous le montre également le graphique 2 .

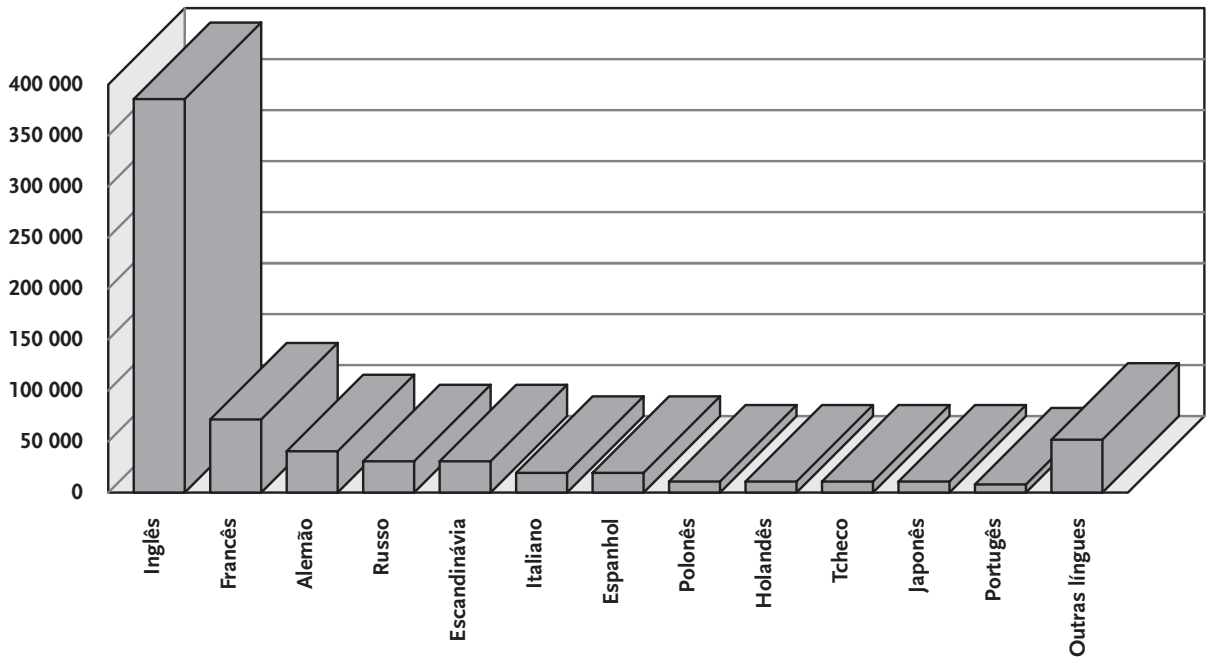

Nous ferons pour l'instant deux remarques à propos de ces données:

- Que les langues les plus traduites du monde correspondent aux littératures les plus traduites du monde, ce qui signifie que le volume de littérature traduite dans chacune de ces langues est supérieur à tous les autres domaines ou genres traduits.

- Que la littérature de langue portugaise représente seulement 1\% de la littérature traduite dans le monde; ajoutons que, toujours selon les données de l'Unesco au cours de la même période, le total des traductions faites en langue portugaise atteint 44744 titres, soit $65 \%$ traduits au Brésil, $29 \%$ traduits au Portugal et $6 \%$ traduits dans les autres pays lusophones. Le Brésil est donc le pays de langue portugaise qui traduit le plus (plus du double du Portugal).

Il s'agit maintenant de savoir quelles sont les langues/cultures qui traduisent le plus dans le monde (tableau 3). C'est ce que Pascale Casanova appelle, en se référant aux langues «défavorisées », l'intraduction, c'est-à-dire, l'importation de textes littéraires étrangers sous la forme de traduction (Casanova 1999: 189). 
TABLEAU 3

Volume de titres des langues/cultures qui traduisent le plus dans le monde (toutes disciplines confondues) de 1970 à 2003

\begin{tabular}{|c|c|c|c|}
\hline Langues & Volume & $\begin{array}{c}\text { Taxinomie des langues } \\
\text { les plus traduites } \\
\text { dans le monde }\end{array}$ & Pourcentage \\
\hline Allemand & 217483 & 1 & $17 \%$ \\
\hline Espagnol & 163036 & 2 & $12 \%$ \\
\hline Français & 133449 & 3 & $10 \%$ \\
\hline Anglais & 91496 & 4 & $7 \%$ \\
\hline Japonais & 78162 & 5 & $6 \%$ \\
\hline Néerlandais & 63712 & 6 & $5 \%$ \\
\hline Russe & 59578 & 7 & $5 \%$ \\
\hline Portugais & 44744 & 8 & $3 \%$ \\
\hline Polonais & 38654 & 9 & $3 \%$ \\
\hline Danois & 38135 & 10 & $3 \%$ \\
\hline Italien & 35927 & 11 & $3 \%$ \\
\hline Norvégien & 27733 & 12 & $2 \%$ \\
\hline TOTAL & 992109 & & \\
\hline Autres langues & 322234 & & $24 \%$ \\
\hline TOTAL GÉNÉRAL & 1314343 & & $100 \%$ \\
\hline
\end{tabular}

L'allemand est la langue/culture qui importe le plus de textes étrangers en les traduisant avec $17 \%$ du total, ce qui peut être interprété par le fait que l'Allemagne est ouverte aux connaissances étrangères et que traduire lui permet de les annexer et/ ou de les diffuser.

GRAPHIQUE 3

\section{Línguas/Culturas que mais traduziram de 1970 a 2003}

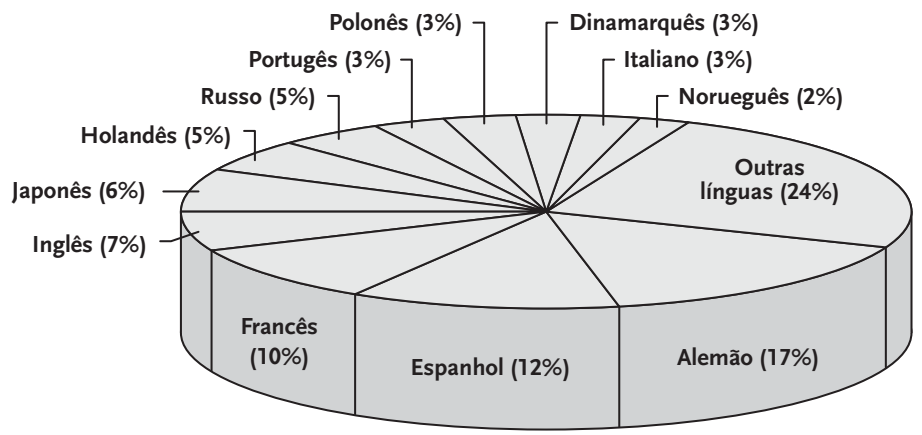

Si l'on confronte ces données des langues qui traduisent le plus avec celles des langues les plus traduites dans le monde, nous observons que: 
- Les livres de langue anglaise sont les plus traduits (52\%) mais d'un autre côté, les pays de langue anglaise traduisent en revanche très peu $(7 \%)$ : ils diffusent et exportent leurs livres, leurs idéologies, leurs cultures, leur savoir mais ils importent très peu les cultures étrangères, ce qui, en réalité, ne confirme que ce que nous savions déjà, soit qu'il s'agit de marchés éditoriaux refermés sur eux-mêmes;

- Les pays/cultures de langue française et allemande ont une politique différenciée, car hormis le fait qu'ils appartiennent aux langues les plus traduites du monde $\left(2^{\mathrm{e}}\right.$ et $3^{\text {e }}$ places), ils importent des livres étrangers en les traduisant; ils sont donc plus ouverts à la traduction, ou plutôt à l'intraduction.

D'autres remarques pourraient être avancées mais pour ce qui nous occupe ici, l'important est de situer les langues et cultures de langues française et portugaise. D'ailleurs, en ce qui concerne la traduction au Brésil, analysons les données de son volume d'intraduction.

Il s'agit ici de quantifier les titres qui ont été traduits au Brésil dans chaque discipline de 1970 à 2003 (tableau 4).

TABLEAU 4

Volume d'intraduction au Brésil (toutes langues confondues)

\begin{tabular}{|l|r|r|}
\hline Littérature & 11725 & $40 \%$ \\
\hline Ouvrages généraux/bibliographies & 306 & $1 \%$ \\
\hline Philosophie/psychologie & 3200 & $11 \%$ \\
\hline Religion/théologie & 3502 & $12 \%$ \\
\hline Droit/sciences sociales/éducation & 2635 & $9 \%$ \\
\hline Sciences naturelles et exactes & 733 & $3 \%$ \\
\hline Sciences appliquées & 4224 & $15 \%$ \\
\hline Arts/jeux/sports & 733 & $3 \%$ \\
\hline Histoire/géographie/biographie & 1765 & $6 \%$ \\
\hline TOTAL & 28863 & $100 \%$ \\
\hline
\end{tabular}

Avec 28863 titres traduits, le Brésil a traduit seulement $2 \%$ du total des traductions dans le monde de 1970 à 2003. Les chiffres indiquent également que ce qui se traduit le plus au Brésil est la littérature (40\%), en accord avec la tendance mondiale qui est de $49 \%$. Ces données apparaissent plus clairement dans le graphique 4 .

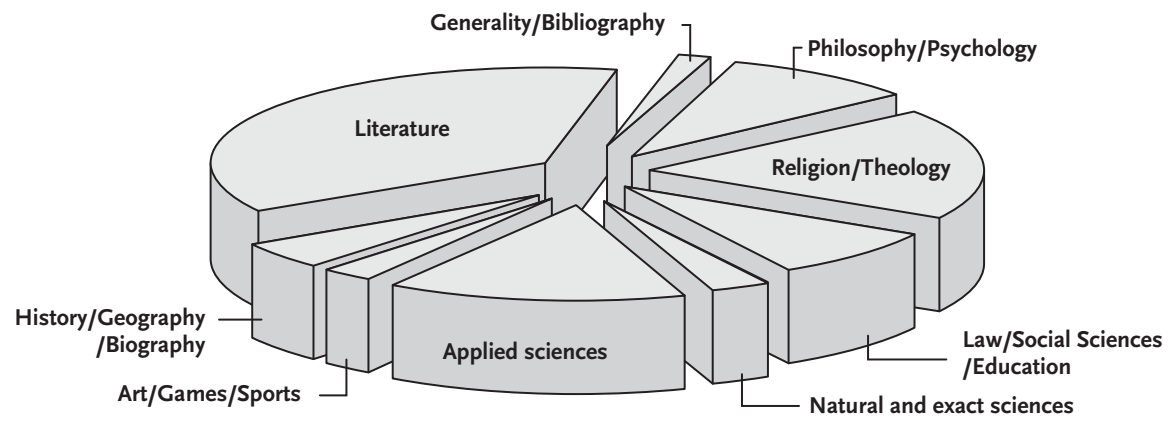


Examinons la littérature traduite au Brésil, en chiffres, décennie par décennie. Selon une approche en diachronie, la littérature traduite se présente de la manière suivante:

- 1970-1979 (758 titres de littérature traduite) $:$ de l'anglais $=494$, de l'allemand $=118$, du français $=94$, de l'espagnol $=27$, de l'italien $=12$ et d'autres langues $=13$.

- 1980-1989 (6 643 titres de littérature traduite) $:$ de l'anglais $=4$ 850, du français $=734$, de l'allemand $=579$, de l'espagnol $=263$, de l'italien $=111$ et d'autres langues $=106$.

- 1990-1999 (4 317 titres de littérature traduite) : de l'anglais $=3$ 382, du français $=337$, de l'allemand $=222$, de l'espagnol $=179$, de l'italien $=114$ et d'autres langues $=83$.

Les traductions issues de la langue française sont très loin derrière celles issues de l'anglais, ce qui ne correspond pas tout à fait aux politiques de diffusion de la francophonie. D'ailleurs, le volume de traduction littéraire par langue nous le confirme (tableau et graphique 5).

TABLEAU 5

\begin{tabular}{|l|c|c|c|}
\hline Langues & Volume & $\begin{array}{c}\text { Taxinomie des langues } \\
\text { les plus traduites } \\
\text { au Brésil }\end{array}$ & Pourcentage \\
\hline Anglais & 8726 & 1 & $74 \%$ \\
\hline Français & 1170 & 2 & $10 \%$ \\
\hline Allemand & 920 & 3 & $8 \%$ \\
\hline Espagnol & 469 & 4 & $4 \%$ \\
\hline Italien & 237 & 5 & $2 \%$ \\
\hline Autres langues & 207 & & $2 \%$ \\
\hline TOTAL & 11725 & & $100 \%$ \\
\hline
\end{tabular}

GRAPHIQUE 5

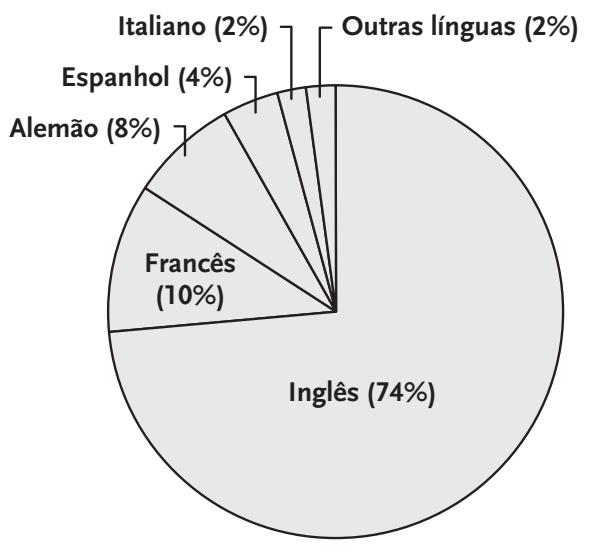

La littérature de langue anglaise est traduite au Brésil sept fois et demie plus que la littérature de langue française, ce qui est énorme. Une analyse rapide et superficielle du corpus des traductions littéraires issues du français nous montre que la littérature pour enfants, et les romans policiers (allant des plus populaires comme les San Antonio ou plus classiques comme ceux du Belge Simenon) représentent la plus 
grande partie de ce qui est traduit. Jules Verne est l'un des auteurs les plus traduits, bien souvent dans des collections pour adolescents, Le petit prince de Saint-Exupéry est le livre qui est le plus réédité avec une quarantaine de rééditions aux éditions Agir à Rio de Janeiro dans une traduction de Marcos Barbosa et les bandes dessinées (Tintin et Astérix) représentent une bonne partie du total des traductions issues du français. Quant aux littératures canadienne et africaine de langue française traduites aux Brésil, elles ne figurent pas dans le corpus de l'Unesco, alors que nous savons que quelques titres d'auteurs canadiens comme Anne Hébert ou Margaret Atwood (de l'anglais) ont été traduits, ainsi qu'une demi-douzaine d'anthologies grâce aux éditions de l'Université fédérale de Rio Grande do Sul.

\section{LE FUTUR: relancer la traduction d'œuvres littéraires françaises et de langue française au Brésil}

Rappelons ce que nous disions en introduction, soit que, comme l'affirme Casanova, «la traduction est la grande instance de consécration spécifique de l'univers littéraire» (1999: 188), et que, donc, la littérature de langue française et française - littérature centrale de l'univers littéraire mondial - devrait être reconnue comme une grande littérature au Brésil et non pas comme une littérature «seconde» (avec $10 \%$ des traductions), loin derrière la littérature de langue anglaise avec $74 \%$ du total des œuvres littéraires traduites au Brésil comme nous l'avons vu.

Les acteurs du marché du livre tant au Brésil qu'en France devraient intervenir pour que l'image littéraire et culturelle de la littérature française traduite au Brésil ne soit pas si déformée. Ainsi, notre contribution tend à montrer que le Brésil, tant qu'il sera exclu du centre, ne fera pas partie des cultures privilégiées par la France pour y diffuser sa langue et sa culture, donc son approche du monde, ses idéologies. Mais il se trouve qu'il n'y a pas une littérature de la "lusophonie» dans le sens où il existe une littérature du commonwealth ou de la francophonie. C'est également peut-être un handicap supplémentaire pour le repositionnement de la littérature brésilienne sur la carte mondiale des littératures et pour reconquérir l'intérêt français et francophone dans la diffusion de leur littérature et de leur culture.

Une dernière question, d'importance, pour conclure: à quoi sert la littérature? On pourrait y répondre en quatre points principaux :

1. Il convient d'analyser d'abord le rapport de l'expérience littéraire au langage: dans quelle mesure l'expérience littéraire est-elle dépendante du langage? Ne se définit-elle pas justement par l'instauration d'un certain rapport au langage? Langue et littérature sont donc intimement liées, et diffuser la littérature reviendrait à diffuser également la langue;

2. Le second point concerne l'enracinement du texte littéraire dans les intentions de son auteur et dans une communauté dont il assumerait les revendications identitaires. Il s'agit ici du rapport, dans le texte littéraire, entre construction de l'identité, reconnaissance du même, et accueil de l'autre, expérience d'autrui en tant qu'il m'est toujours quelque part étranger. La littérature diffuse donc l'autre en tant que différent et non en tant que même;

3. Ensuite, le troisième point est celui de l'accès au monde que nous procure le texte littéraire - autrement dit, de son caractère cognitif ou non cognitif;

4. Enfin, le quatrième et dernier point concerne le rapport du texte littéraire aux valeurs: en quoi le texte littéraire contribue-t-il à l'expérience et la transmission des valeurs? 
Les questions soulevées par le rôle et la fonction de la littérature, et a fortiori par la littérature traduite, sont multiples et de taille: problème de la relation entre le texte littéraire et le contexte politique, social, culturel, de sa production et de ses réceptions; problème de la capacité du texte littéraire, et notamment du texte fictionnel, à représenter le réel; problème de la définition même de la littérature et de la valeur littéraire.

Le futur dépend donc des stratégies politiques, culturelles, éditoriales et commerciales des biens culturels qui peuvent faire évoluer le volume de traductions littéraires issues du français quantitativement et qualitativement et dépend aussi de nous, agents diffuseurs non seulement des littératures, mais encore des langues et des cultures.

\section{NOTES}

1. Cet article est issu d'une communication présentée lors du XIV ${ }^{e}$ Congrès brésilien des professeurs de français en août 2003.

2. Nous revenons à la question de l'espace interculturel dans notre partie «Les traducteurs» (Chapitre 2).

3. Notre parcours académique (une licence puis un «master» en littérature brésilienne de l'Université fédérale de Florianópolis, au Brésil), notre parcours de vie (habitant depuis 13 ans le Brésil), notre parcours futur (retour à l'enseignement à l'Université).

4. Mário Carelli, chargé de recherche au CNRS, était également agrégé de portugais et traducteur de romans brésiliens.

5. Il a aussi fait des conférences à Anvers (18-25 janvier 1909) et d'autres à Paris (15 mars au 6 mai 1911).

6. OBS: Le directeur de l'École normale nupérieure proposa à Claude Lévi-Strauss de poser sa candidature comme professeur de sociologie à l'Université de São Paulo. Et, connaissant son attirance pour l'ethnographie, il ajouta «les faubourgs sont remplis d'Indiens, vous leur consacrerez vos week-ends». Propos non fondés, comme l'explique Lévi-Strauss (1955:47), puisqu'il découvrira à São Paulo un gigantesque centre urbain.

7. Hongrois: 9 440; Japonais: 7 039; Arabe: 7 024; Portugais: 5 836; Chinois: 5075.

\section{RÉFÉRENCES}

Carelli, M. (1987) : «Interactions culturelles franco-brésiliennes», dans France-Brésil: bilan pour une relance, Paris, Entente.

Carelli, M. (1993) : «La France Antarctique et la France Equinoxiale, espaces d'utopie », dans La rencontre des imaginaires entre Europe et Amériques, Luc Bureau, Jean Ferrari, Jean-Jacques Wunenburger (dirs.), Paris, L'Harmattan.

Casanova, P. (1999): La République Mondiale des Lettres, Paris, Seuil, "Prière-d'insérer».

LAmbert, J. (1989): "La traduction, les langues et la communication de masse», In Target $1: 2$, International Journal of Translation Studies, Amsterdam, Benjamins.

Lambert, J. (1990): "À la recherche de cartes mondiales des littératures», dans Semper Aliquid Novi, Janos Riesz et Alain Ricard (dir.), Tübingen, Narr.

Lambert, J. et D. Delabastita (1996): «La Traduction de textes audiovisuels: modes et enjeux culturels », dans Les Transferts linguistiques dans les médias audiovisuels, Yves Gambier (dir.), Lille, Presses universitaires de Lille, p. 3-58.

Lévi-Strauss, C. (1955): Tristes tropiques, Paris, Plon.

PyM, A. (1998): Method in Translation History, Manchester, Saint Jerome.

Valezi Staut, L. M. (1991): A recepção da obra machadiana na França, São Paulo, thèse de doctorat non publiée. 